\title{
Simulation reductions for the Ising model
}

\author{
Version: October 24, 2018
}

\author{
Mark L. Huber \\ Department of Mathematics and Computer Science, Claremont McKenna College \\ mhuber@cmc.edu
}

\begin{abstract}
Polynomial time reductions between problems have long been used to delineate problem classes. Simulation reductions also exist, where an oracle for simulation from some probability distribution can be employed together with an oracle for Bernoulli draws in order to obtain a draw from a different distribution. Here linear time simulation reductions are given for: the Ising spins world to the Ising subgraphs world and the Ising subgraphs world to the Ising spins world. This answers a long standing question of whether such a direct relationship between these two versions of the Ising model existed. Moreover, these reductions result in the first method for perfect simulation from the subgraphs world and a new Swendsen-Wang style Markov chain for the Ising model. The method used is to write the desired distribution with set parameters as a mixture of distributions where the parameters are at their extreme values.
\end{abstract}

\section{Introduction}

In this paper, two different forms of the Ising model are linked via a polynomial time simulation reduction. Consider a family of probability distributions $\pi$ parametrized by inputs $\mathcal{I}$, together with another family $\pi^{\prime}$. Say that $\pi$ is simulation reducible to $\pi^{\prime}$ if an algorithm exists for drawing from $\pi$ on $\mathcal{I}$ that is allowed to take draws from $\pi^{\prime}$ as well as utilize extra randomness in the form of independent Bernoulli draws whose parameter is determined at runtime. In other words, if the ability to draw from $\pi^{\prime}$ together with the ability to flip coins with arbitrary probabilities of coming up heads is enough to draw a sample from $\pi$, then $\pi$ is simulation reducible to $\pi^{\prime}$. As with problem reductions, sampling reductions are of most interest when they are polynomial, that is, when the sum of the sizes of the inputs to $\pi^{\prime}$ plus the number of Bernoullis used is a polynomial in the size of $\mathcal{I}$.

A famous example of simulation reductions appears in the Swendsen-Wang approach [9] to the Ising model. The original version of the Ising model used spins on nodes in graphs (call this the spins view) while another used subsets of edges in graphs (call this the random cluster view). As part of the Swendsen-Wang algorithm, a draw from the random cluster model could be turned (using a number of Bernoullis equal 
to the number of clusters formed by the edges) into a spins draw. Similarly, a spins draw could be turned (using a number of Bernoullis equal to the number of edges in the graph) into a random cluster draw.

In this work, a third view of the Ising model is dealt with. This third view also uses subsets of edges, but assigns them weights that are very different from those of the random cluster model. Following [3], this third model will be called the subgraphs view of the Ising model.

The families of distributions corresponding to spins, random clusters, and subgraphs will be denoted $\pi_{\text {spins }}, \pi_{\text {rc }}$, and $\pi_{\text {subs }}$ respectively. The remainder of the paper is organized as follows. In Section 2, the three families of the Ising model are described in detail. Section 3 discusses the framework that will be used for the reductions. Section 4 shows how a single draw from the subgraphs model together with a number of Bernoullis at most equal to the number of edges can be used to create a draw from the random cluster model. Section 5 then shows the reverse direction: how a single draw from the random cluster model together with a number of Bernoullis equal to the number of edges can be used to draw from the subgraphs model. Since the reductions between $\pi_{\text {rc }}$ and $\pi_{\text {spins }}$ are well known, and require a single sample each, it is possible to take a single draw from $\pi_{\text {subs }}$ and convert it to a single draw from $\pi_{\text {spins }}$ with a small number of Bernoullis. Similarly, a single draw from $\pi_{\text {spins }}$ can be converted to a single draw from $\pi_{\text {subs }}$ with a small number of Bernoullis. This yields a new Swendsen-Wang style Markov chain for approximately sampling from the Ising model. Also, it answers a long standing question in [3] of whether or not such a direct relationship between the distributions $\pi_{\text {spins }}$ and $\pi_{\text {subs }}$ existed. These results are discussed in detail in Section 6 .

Earlier work in [7] created an approximate simulation reduction from $\pi_{\text {spins }}$ to $\pi_{\text {subs }}$ by creating a series of approximations of partition functions for multiple inputs. This method has the drawback of requiring multiple draws from $\pi_{\text {subs }}$ in order to obtain a single configuration that is approximately drawn from $\pi_{\text {spins. }}$. In contrast, the reduction presented here is exact, and requires only one draw from $\pi_{\text {subs }}$ to generate one draw from $\pi_{\text {spins }}$ or vice versa. Because the reduction is exact, this provides for the first perfect simulation algorithm for $\pi_{\text {subs }}$, the details are also in Section 6 .

\section{The Ising model}

The Ising model was initially proposed as a model for magnitization and has been studied extensively becuase of the presence of a phase transition in lattices of dimension two or higher [8]. It has also been employed in statistical applications including agricultural studies and image restoration [1].

Spins model In the ferromagnetic Ising model each node of a graph $G=(V, E)$ is assigned either a 1 or -1 . Because of the original use as a model of magnetism, the 1 nodes are referred to as "spin up" and the -1 nodes are called "spin down".

A state $x \in\{-1,1\}^{V}$ is called a configuration. The weight of a configuration is of the form:

$$
w_{\text {spins }}(x)=\prod_{\{i, j\} \in E} f(x(i), x(j))
$$


For a weight function $w_{\text {spins }}(x)$, the probability distribution is $\pi_{\text {spins }}(x)=w_{\text {spins }}(x) / Z_{\text {spins }}$, where $Z_{\text {spins }}=\sum_{x \in\{-1,1\} V} w_{\text {spins }}(x)$ is called the partition function.

Let $\beta$ be a nonnegative vector over the edges $E$, and $B$ be a nonnegative vector over the nodes $V$. Then

$$
f(x(i), x(j))= \begin{cases}\exp (\beta(\{i, j\}) x(i) x(j)) & \beta(\{i, j\})<\infty \\ \mathbf{1}(x(i)=x(j)) & \beta(\{i, j\})=\infty\end{cases}
$$

Hence $\beta$ controls the strength of interaction between spins at endpoints of edges. When $\beta$ is infinite, the attraction is infinite, and endpoints must be given the same value.

An extension of the basic Ising model is to allow for an external magnetic field. In this case, the weight function acquires factors based directly on the value of each node:

$$
w_{\text {spins+field }}(x)=\left[\prod_{\{i, j\} \in E} f(x(i), x(j))\right]\left[\prod_{i \in V} g(x(i))\right],
$$

where $g$ is paramerized by a vector $B$ that controls the strength of the magnetic field:

$$
g(x(i))=\left\{\begin{array}{lll}
\exp (B(i) x(i)) & \text { for } & |B(i)|<\infty \\
\mathbf{1}(x(i)=1) & \text { for } & B(i)=\infty \\
\mathbf{1}(x(i)=-1) & \text { for } & B(i)=-\infty
\end{array}\right.
$$

Say that the magnetic field is unidirectional if $B \geq 0$ or $B \leq 0$. The unidirectional model is easier than the model with general magnetic field in the sense that it can be easily reduced to the case with no magnetic field as follows. Suppose without loss of generality $B \geq 0$.

If $B(v)<\infty$ for all $v$, then build a new graph by creating a dummy node $v_{B}$ and for all $i \in V$ adding an edge $\left\{i, v_{B}\right\}$ with edge weight $\beta\left(\left\{i, v_{B}\right\}\right)=(1 / 2) B(i)$. Note that any configuration $x$ satisfying $x\left(v_{B}\right)=1$ has

$$
w_{\text {spins }}+\text { field }(x(V))=w_{\text {spins only }}(x(V)) \prod_{i \in V: B(i)<\infty} \exp ((1 / 2) B(i)) .
$$

Since the weights differ by only a constant, the probability distributions are the same.

Moreover, under $w_{\text {spins only }}$ the weight of $x$ and $-x$ are the same, so taking a draw from $w_{\text {spins }}$ and choosing $x$ or $-x$ so that $v_{B}$ has value 1 is an easy way to draw from $w_{\text {spins }}$ conditioned on $v_{B}$ being 1 . Hence the unidirectional magnetic field can be brought into the case of no magnetic field with no loss of generality when $B(v)<\infty$ for all $v$.

When there exist one or more nodes with $B(v)=\infty$, there is no need to create the dummy node $v_{B}$. These nodes all must be spin up, and so they can be merged, and then the merged node can be used in the same fashion as $v_{B}$ above.

Subgraphs model In a seminal paper Jerrum and Sinclair [3] showed how to approximate $Z_{\text {spins }}(\beta)$ for any graph and any $\beta$ in polynomial time. Their approach did not tackle the spins model directly. Instead, they developed results for a different formulation of the Ising model: the subgraphs model. This model is also a Markov random field, but assigns values to the edges of the graph rather than the nodes. 
Let $\Omega_{\text {subs }}=\{0,1\}^{E}$, and now a configuration $y \in \Omega_{\text {subs }}$ can be viewed as a collection of edges of the graph, where $y(e)=1$ indicates that the edge is in the collection and otherwise it is not. Since each $y$ encodes a subset of edges, this was called the subgraphs world in [3]. Define $\lambda$ over the edges as follows:

$$
\lambda(\{i, j\})=\tanh \beta(\{i, j\})
$$

where $\tanh \infty$ is taken to be 1 . Then in the subgraphs model, the weight function is

$$
w_{\text {subs }}(y)=\left[\prod_{e: y(e)=1} \lambda(e)\right]\left[\prod_{i} \mathbf{1}(\operatorname{deg}(i, y) \text { is odd })\right] .
$$

The degree of a node $i$ in subgraph configuration $y$ is

$$
\operatorname{deg}(i, y):=\sum_{j \neq i} y(\{i, j\})
$$

and $\mathbf{1}(A)$ is the indicator function that has value 1 if the Boolean expression $A$ is true, and is 0 otherwise.

As with the spins model, $\pi_{\text {subs }}(y)=w_{\text {subs }}(y) / Z_{\text {subs }}$, where $Z_{\text {subs }}$ is the sum of the weights over all configurations, or equivalently the normalizing constant that makes $\pi_{\text {subs }}$ a probability distribution. The subgraphs model is also known as the high temperature expansion, and was introduced by van der Waerden [10. Like the spins model, here the weight depends on the product of individual factors that depend either on a single edge or only on the edges leaving a particular node.

One note: in the Jerrum and Sinclair [3] paper the formulation of the subgraphs world included terms that allowed for a unidirectional magnetic field. As noted in the previous section, a unidirectional field can be eliminated from the problem without loss of generality, and so here the simpler form of the subgraphs model is used.

Random Cluster model A third approach to the Ising model was introduced by Fortuin and Kasteleyn [2]. Unlike the spins and subgraphs views of the Ising model, the random cluster model is decidedly nonlocal in its weight function.

Like the subgraphs model, the state space $\Omega_{\mathrm{rc}}=\{0,1\}^{E}$ indexes a collection of edges. These edges partition the nodes $V$ into the set of maximally connected components, known as clusters. To be precise, consider a configuration $z \in \Omega_{\mathrm{rc}}$. A collection of nodes $C$ is a cluster in $z$ if for all $v$ and $v^{\prime}$ in $C$, there is a path $\left\{v=v_{1}, v_{2}, v_{3}, \ldots, v_{n}=v^{\prime}\right\}$ such that $\left\{v_{i}, v_{i+1}\right\} \in E$ and $z\left(\left\{v_{i}, v_{i+1}\right\}\right)=1$ for all $i$. Moreover, for all $v \in C$ and $v^{\prime \prime} \notin C$, either $\left\{v, v^{\prime \prime}\right\} \notin E$ or $z\left(\left\{v, v^{\prime \prime}\right\}\right)=0$. Let $\mathcal{C}$ denote the set of clusters.

The weight function for the random cluster model is:

$$
w_{\mathrm{rc}}(z)=\left[\prod_{e: z(e)=1} p(e)\right]\left[\prod_{e: z(e)=0}(1-p(e))\right] 2^{\# \mathcal{C}} .
$$

where

$$
p(e)=1-\exp (-2 \beta(e))
$$


and $\exp (-\infty)$ is taken to be 0 .

The procedure for generating a spins draw from a random cluster draw is as follows: independently for each cluster, draw uniformly from $\{-1,1\}$ and assign all nodes in that cluster the randomly chosen value. (See [9] for further details.)

The idea behind this is as follows. The spins model with parameter $\beta$ can be viewed as a mixture of many different spins models, each with a different set of parameters. Each component of the mixture has a parameter vector whose $\beta$ values for every edge are either 0 or $\infty$, and so altogether there are $2^{\# E}$ different components of the mixture. The random cluster configuration indexes which component of the mixture to use: $z(e)=1$ indicates $\beta(e)=\infty$, while $z(e)=0$ indicates $\beta(e)=0$. Drawing a spins model with such a simple parameter vector is easy: all nodes connected by edges with $\beta(e)=\infty$ must have the same spin, while the $\beta(e)=0$ edges might as well be gone from the graph.

This idea of using an index from a mixture is developed formally in Section 3 .

Relationship between spins and subgraphs The normalizng constants $Z_{\text {spins }}$ and $Z_{\text {subs }}$ are related by an easy to calculate constant. The following result goes back to [5].

Theorem 1.

$$
Z_{\text {spins }}=Z_{\text {subs }} 2^{\# V} \prod_{e} \cosh \beta(e) .
$$

While this result is straightforward to show analytically, such a proof does not offer much probabilistic insight into why this remarkable relationship is true. In [3] it is noted that "there is no direct correspondence between configurations in the two domains and the subgraph configurations have no obvious physical significance". In Section 6 a new proof of this result is presented that is based on the simulation reductions presented here.

In [3], a Markov chain that moved among configurations in the subgraphs world was developed. More importantly, this chain was shown to be rapidly mixing for all graphs. This result could then be used with the idea of selfreducibility [4] to obtain a fully polynomial approximation scheme (fpras) for $Z_{\text {subs }}$, and hence $Z_{\text {spins }}$ as well.

\section{Drawing from mixtures}

The reductions between spins and subgraphs draws come from viewing a distribution $\pi$ as a convex mixture of several distributions, so

$$
\pi=\alpha_{1} \pi_{1}+\cdots \alpha_{M} \pi_{M}
$$

where $\alpha_{1}+\cdots \alpha_{M}=1$.

Typically the parameters for distributions of interest (like the Ising model) form a convex set. The mixture of $\pi$ uses distributions where the parameter values are at their extreme values. Usually these extreme values of parameters correspond to simpler

distributions. Many times, in reducing from $\pi$ to $\pi^{\prime}, \pi^{\prime}=\alpha_{1} \pi_{1}^{\prime}+\cdots \alpha_{M} \pi_{M}^{\prime}$ where the $\alpha_{i}$ are equal to the cofficients for $\pi$. Therefore, the algorithm proceeds as follows: 
(1) Draw a sample from $\pi$,

(2) Choose which $\pi_{i}$ the sample came from,

(3) Draw a sample from $\pi_{i}^{\prime}$,

(4) Return as a sample from $\pi^{\prime}$.

This can be formulated as a special case of the auxilliary variable method. Let $I$ be a random variable where $\mathbb{P}(I=i)=\alpha_{i}$ (this extra variable $I$ is the auxilliary variable.) If $X \mid I \sim \pi_{I}$, then $X \sim \pi$. The algorithm takes advantage of this in reverse: Given $X \sim \pi$ begin by choosing $I \mid X$. Then choose $X^{\prime} \mid I$ from $\pi^{\prime}$. Then $X^{\prime} \sim \pi^{\prime}$ as desired.

Typically, direct computation of the $\alpha_{i}$ is difficult, which is why it is not possible to just draw a random $I$ directly. However, drawing $I \mid X$ is possible, by proceeding through several stages.

Begin by considering a mixture of two distributions, so $\pi=\alpha_{1} \pi_{1}+\alpha_{2} \pi_{2}$. At this first stage, choose $I(1) \in\{1,2\}$ given $X$. Then break $\pi_{I(1)}$ into two distributions, and so on until the index $I=(I(1), I(2), \ldots, I(k))$ has been chosen. Typically the stages are set up so that $\pi_{I}^{\prime}$ is easy to sample from. The following theorem provides the algorithm for choosing $I(a)$ given $X$ from the two choices.

Theorem 2. Consider three distributions $\pi, \pi_{1}$ and $\pi_{2}$, respectively defined by unnormalized weight functions $w, w_{1}$ and $w_{2}$ with the appropriate normalizing constants $Z=$ $\sum_{y} w(y), Z_{1}=\sum_{y} w_{1}(y)$, and $Z_{2}=\sum_{y} w_{2}(y)$. Suppose that $w(x)=c_{1} w_{1}(x)+c_{2} w_{2}(x)$, where the $c_{i}$ are positive constants.

Then $\pi$ is a mixture of $\pi_{1}$ and $\pi_{2}$, so $\pi=\alpha_{1} \pi_{1}+\alpha_{2} \pi_{2}$. Let $X \sim \pi$. Let $I \mid X$ be a binary random variable that is 1 with probability $c_{1} w_{1}(X) /\left[c_{1} w_{1}(X)+c_{2} w_{2}(X)\right]$ and is 2 otherwise. Then $\mathbb{P}(I=1)=\alpha_{1}, \mathbb{P}(I=2)=\alpha_{2}$, and $[X \mid I] \sim \pi_{I}$.

Proof. Start with $w(x)=c_{1} w_{1}(x)+c_{2} w_{2}(x)$ and divide through by $Z$. This yields $\pi(x)$ on the left hand side. Multiply and divide term $i$ on the right hand side by $Z_{i}$ to obtain:

$$
\pi(x)=c_{1} \pi_{1}(x)\left(Z_{1} / Z\right)+c_{2} \pi_{2}(x)\left(Z_{2} / Z\right) .
$$

Hence $\alpha_{1}=c_{1} Z_{1} / Z$ and $\alpha_{2}=c_{2} Z_{2} / Z$. Also,

$$
\mathbb{P}(I=i)=\sum_{y} \mathbb{P}(I=i \mid X=y) \pi(y)=\sum_{y} \frac{c_{i} w_{i}(y)}{w(y)} \frac{w(y)}{Z}=\frac{c_{i}}{Z} \sum_{y} w_{i}(y)=\frac{c_{i} Z_{i}}{Z}=\alpha_{i} .
$$

Turning it around:

$$
\mathbb{P}(X=y \mid I=i)=\mathbb{P}(I=i \mid X=y) \pi(y) / \alpha_{i}=\frac{c_{i} w_{i}(y)}{w(y)} \frac{w(y)}{Z} \frac{Z}{c_{i} Z_{i}}=\frac{w_{i}(y)}{Z_{i}} .
$$

So $[X \mid I=i]$ has distribution $\pi_{i}$, completing the proof.

This theorem can be easily extended to an arbitrary number of distributions, however, mixtures of $\pi_{1}$ and $\pi_{2}$ suffice for all the results in this paper. 


\section{Subgraphs to random cluster}

In this section it is shown how to utilize a subgraphs draw together with at most \#E Bernoulli draws to generate a draw from the random cluster model. This can then be used to create a spins draw if desired.

Let $\pi_{\text {subs }}$ be the distribution parameterized by $\lambda$. Consider any edge $e$ with $\lambda(e) \in$ $(0,1)$. Create a new vector $\lambda_{e \rightarrow 1}$ that takes on value 1 on edge $e$, and matches $\lambda$ at all other edges. Similarly, let $\lambda_{e \rightarrow 0}$ equal $\lambda$ on all edges but $e$, and let $\lambda_{e \rightarrow 0}(e)=0$.

Then since $\lambda(e) \in(0,1)$ :

$$
w_{\text {subs }}(y ; \lambda)=\lambda(e) w_{\text {subs }}\left(y ; \lambda_{e \rightarrow 1}\right)+(1-\lambda(e)) w_{\text {subs }}\left(y ; \lambda_{e \rightarrow 0}\right)
$$

This has the exact form needed for Theorem 2, The algorithm is simple. Begin with a draw $Y$ from $\pi$ parameterized with $\lambda$. Calculate $\lambda(e)$ times the weight of $Y$ under $\lambda_{e \rightarrow 1}$, and $(1-\lambda(e))$ times the weight of $Y$ under $\lambda_{e \rightarrow 0}$. Draw index $I$ from $\{1,0\}$ with probability proportional to these two numbers, then set $\lambda(e)$ to $I$.

This means $\mathbb{P}(I=1)=\lambda(e) w_{\text {subs }}\left(Y ; \lambda_{e \rightarrow 1}\right) / w_{\text {subs }}(Y ; \lambda)$. When $Y(e)=1$, this is just 1. When $Y(e)=0$, this becomes $\lambda(e)$. Therefore

$$
\mathbb{P}(I=1)=\lambda(e)+(1-\lambda(e)) Y(e) .
$$

After choosing the value of $I, Y$ given $I$ comes from the correct half of the mixture, and so the process can be repeated again, until all of the entries in $\lambda$ are either 0 or 1 .

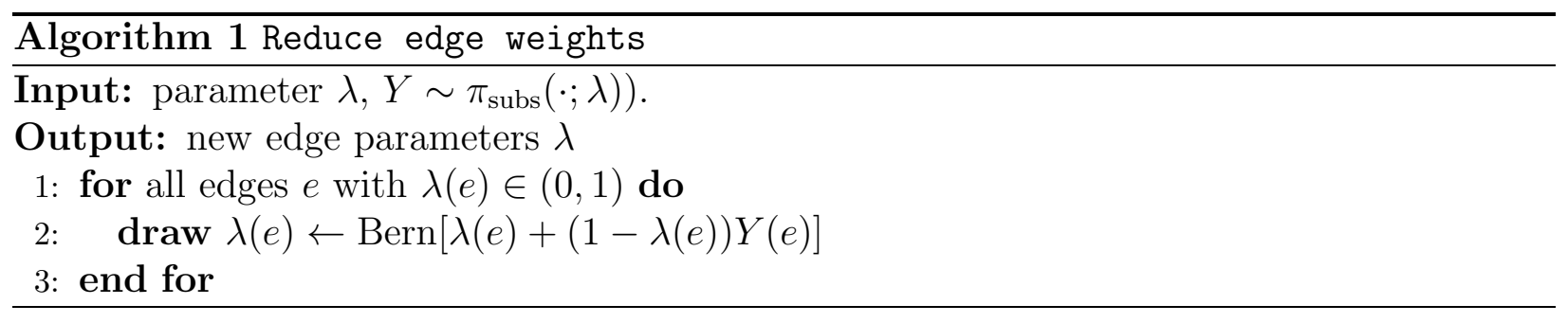

Note that when $Y(e)=1$, then $\lambda(e) w_{\text {subs }}\left(Y ; \lambda_{e \rightarrow 1}\right) / w_{\text {subs }}(Y ; \lambda)=1$, so the algorithm always sets $\lambda(e)$ to 1 in this case. Only when $Y(e)=0$ is there a choice with the possibility of $\lambda(e)=0$.

At the end of the this algorithm, the draw $Y$ has been slotted into one of $2^{\# E}$ components of a mixture indexed by the new parameter vector $\lambda$. Each element of $\lambda$ is now either 0 or 1 , in the spins world this corresponds to $\beta$ being either 0 or $\infty$. Hence the two nodes are either independent of each other or forced to be the same, just as in the random cluster model.

This means, if the random output of Algorithm 1 is called $\Lambda$, then $\Lambda$ is a draw from the random cluster model of Fortuin and Kasteleyn [2]. This is stated precisely in the following theorem.

Theorem 3. Let $Y \sim \pi_{\text {subs }}(\cdot ; \lambda)$. Then let $\Lambda$ be the output of Algorithm 1 with input $(\lambda, Y)$. Let $p(e)=1-\exp (-2 \beta(e))=2 \lambda(e) /(1+\lambda(e))$. Then

$$
\mathbb{P}(\Lambda=z)=\frac{1}{Z_{\mathrm{rc}}}\left[\prod_{e: z(e)=1} p(e)\right]\left[\prod_{e: z(e)=0} 1-p(e)\right] 2^{\# \mathcal{C}(z)}
$$


where

$$
Z_{\mathrm{rc}}=Z_{\mathrm{subs}} 2^{\# V-\# E} \prod_{e}(1+\exp (-2 \beta(e)) .
$$

Proof. Fix $z \in\{0,1\}^{E}$. Consider a particular cluster in $z$. Suppose that $Y=y$, and consider the chance that $\Lambda=z$ given $Y=y$. Edges with $y(e)=1$ always have $\Lambda(e)=1$, so if $z(e)=0$ then $y(e)$ must be zero to have positive chance of $\Lambda=z$. That is, $y \leq z$.

Now consider how a random draw could result in $\Lambda=z$ given $Y=y$. For each edge with $y(e)=0$ and $z(e)=1$, there is a $\lambda(e)$ chance of making this choice. Hence the chance these edges agree is $\prod_{e: y(e)=0, z(e)=1} \lambda(e)$. For each edge $e$ with $y(e)=1$ and $z(e)=1$, the chance these edges agree is 1 . Finally, if $y(e)=0$ and $z(e)=0$, the chances that these agree is $\prod_{e: y(e)=0, z(e)=0} 1-\lambda(e)$. Therefore,

$$
\mathbb{P}(\Lambda=z \mid Y=y)=\left[\prod_{e: y(e)=0, z(e)=1} \lambda(e)\right]\left[\prod_{e: y(e)=0, z(e)=0} 1-\lambda(e)\right]
$$

Now consider the probability that $Y=y$. Since $Y$ is a subgraphs draw, this probability is 0 unless all the nodes under $y$ have even degree, in which case the probability of choosing $y$ is $Z_{\text {subs }}^{-1} \prod_{y(e)=1} \lambda(e)$. This equals $Z_{\text {subs }}^{-1} \prod_{y(e)=1, z(e)=1}$ since $y(e)=1$ implies $z(e)=1$ as well. Therefore for all $y$ where each node has even degree

$$
\begin{aligned}
\mathbb{P}(Y=y, \Lambda=z) & =Z_{\text {subs }}^{-1}\left[\prod_{e: y(e)=1, z(e)=1} \lambda(e)\right]\left[\prod_{e: y(e)=0, z(e)=1} \lambda(e)\right]\left[\prod_{e: y(e)=0, z(e)=0} 1-\lambda(e)\right] \\
& =Z_{\text {subs }}^{-1}\left[\prod_{e: z(e)=1} \lambda(e)\right]\left[\prod_{e: z(e)=0} 1-\lambda(e)\right]
\end{aligned}
$$

To find $\mathbb{P}(\Lambda=z)$ all that remains is to sum over all $y$ where each node has even degree. Since $\mathbb{P}(Y=y, \Lambda=z)$ is independent of $y$,

$$
\mathbb{P}(\Lambda=z)=\#\{y: y \leq z, \operatorname{deg}(i, y) \text { even } \forall i\} Z_{\text {subs }}^{-1}\left[\prod_{e: z(e)=1} \lambda(e)\right]\left[\prod_{e: z(e)=0} 1-\lambda(e)\right]
$$

So now consider how many $y \leq z$ states have even degree everywhere. Consider a particular cluster $C$ in $z$, and let $C_{E}$ denote the set of edges between nodes in $C$. Since the nodes are connected, there exists a tree $T$ with edges $T_{E} \subseteq C_{E}$. Let $A=C_{E} \backslash T_{E}$ be those edges in the cluster that are not part of the tree.

The key fact about $A$ is that for any value of $y(A)$ there exists exactly one value of $y\left(T_{E}\right)$ that ensures that $y\left(T_{E} \sqcup A\right)$ has even degree at each node. See Figure 1 for an example.

To show this, fix $y(A)$. Start with a leaf $i$ of the tree $T$. There is exactly one edge in $T_{E}$ adjacent to $i$ with $z(e)=1$, so there is precisely one way to choose $y(e)$ in order to maintain that the degree of $i$ must be even under $y(e)$. Now consider the remaining 


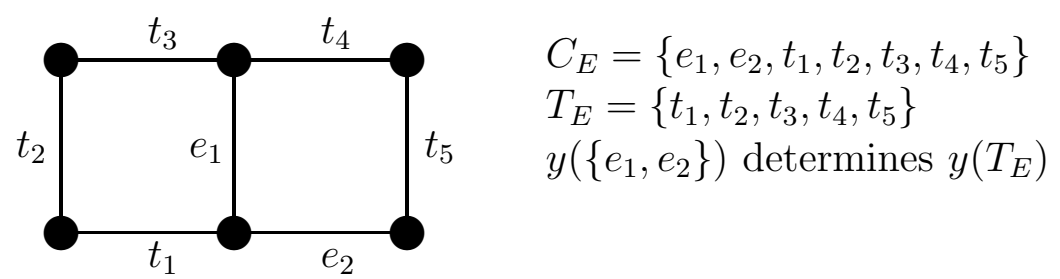

Figure 1: Example of cluster with six nodes

edges in $T_{E}$. The nodes connected to these edges forms a smaller tree, so again a leaf exists, and again the there is exactly one way to choose $x$ on this edge to maintain the even degree requirement. After \#T steps the unique value for $y(T)$ that preserves the even degree at each node will have been found.

So given $y(A)$, there is exactly one choice of $y$ over the edges of the cluster where all edges of the cluster receive even degree. There are $2^{\# A}$ different possible values of $y(A)$, and so there are $2^{\# A}$ different choices of $y$ over the cluster. The number of edges in $A$ is the number of edges in the cluster minus the number of edges in the tree, so for a cluster $C \in \mathcal{C}$, this is

$$
\# A=\left[\sum_{e \in C_{E}} z(e)\right]-[\# C-1]
$$

The choice of $y\left(C_{E}\right)$ is independent for each cluster $C \in \mathcal{C}$, and so the total number of $x$ such that $x \leq z$ and each node of $x$ has even degree is the product

$$
\prod_{C \in \mathcal{C}} 2^{\sum_{e \in C} z(e)-[\# C-1]}=2^{\left[\sum_{e \in C} z(e)\right]-\# V+\# \mathcal{C}(z)} .
$$

Combining this with (8) yields

$$
\mathbb{P}(\Lambda=z)=Z_{\text {subs }}^{-1} 2^{-\# V+\# \mathcal{C}(z)}\left[\prod_{e: z(e)=1} 2 \lambda(e)\right]\left[\prod_{e: z(e)=0} 1-\lambda(e)\right]
$$

or equivalently,

$$
\mathbb{P}(\Lambda=z)=Z_{\text {subs }}^{-1} 2^{-\# V}\left[\prod_{e} 1+\lambda(e)\right] 2^{\# \mathcal{C}(z)}\left[\prod_{e: z(e)=1} \frac{2 \lambda(e)}{1+\lambda(e)}\right]\left[\prod_{e: z(e)=0} \frac{1-\lambda(e)}{1+\lambda(e)}\right] .
$$

It is easy to verify from (2) and (5) that $2 \lambda(e) /(1+\lambda(e))=p(e)$ and $(1-\lambda(e)) /(1+$ $\lambda(e))=1-p(e)$, which shows that the two rightmost sets of brackets factors equal $w_{\mathrm{rc}}(z)$. Therefore $Z_{\text {subs }}^{-1} 2^{-\# V} \prod_{e}(1+\lambda(e))=Z_{\mathrm{rc}}^{-1}$. Using $(1+\lambda(e))^{-1}=(1 / 2)(1+$ $\exp (-2 \beta(e)))$ completes the proof. 


\section{Random cluster to subgraphs}

This section shows how to take a random cluster draw, and together with a number

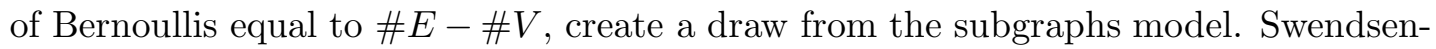
Wang [9] employed the relationship between random clusters and spins to devise a Markov chain that is very fast for some values of the parameters. From a spins draw, generate a random cluster index, then from the index, generate a new spins draw.

With the ability to move between a random cluster draw and a subgraphs draw, a new Swendsen-Wang style Markov chain becomes available for use: from the random cluster model, draw a subgraphs model, then from the subgraphs model, draw a random cluster model. Algorithm 2 shows how to move from a random cluster model to a subgraphs model.

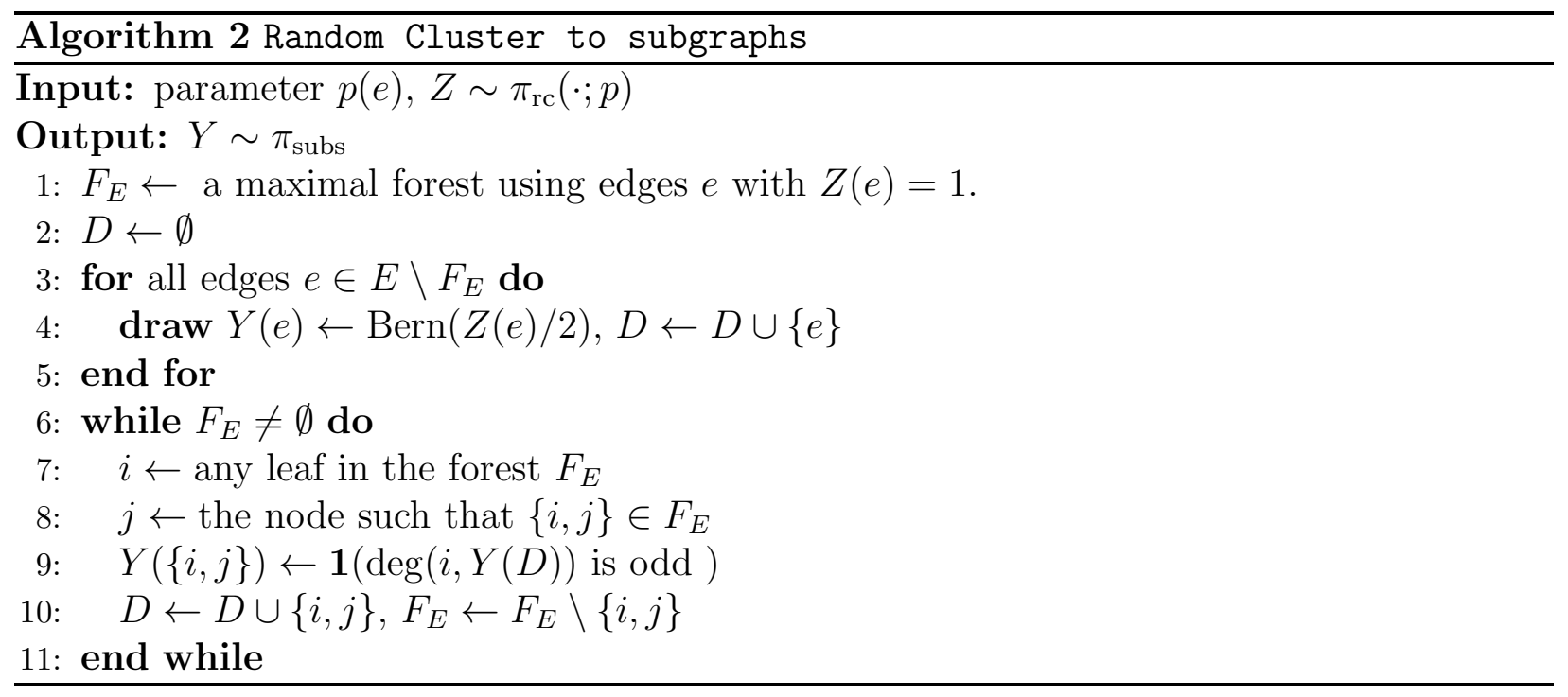

Theorem 4. Algorithm 2 returns a draw from the subgraphs distribution where parameters $\lambda, \beta$ and $p$ are related by equations (2) and (5).

Proof. It was shown in Theorem 3 that if $Y \sim \pi_{\text {subs }}(\cdot ; \lambda)$, and then $Z \mid Y^{\prime}$ is drawn as an index for the component of the mixture, then $Z \sim \pi_{\text {rc }}(\cdot ; p)$ where $p$ and $\lambda$ are related by (2) and (5). So as in the discussion in Section 3, if $Z$ is drawn first from $\pi_{\mathrm{rc}}(\cdot ; p)$, and then $Y \mid Z \sim \pi_{\text {subs }}(\cdot ; Z)$, then $Y \sim \pi_{\text {subs }}(\cdot ; \lambda)$.

Let $F_{E}$ be a maximal spanning forest in the graph using edges with $Z(e)=1$. Any edge not in $F_{E}$ with $Z(e)=0$ must have $Y(e)=0$ in order for the configuration to have positive weight. So let $e$ be an edge such that $Z(e)=1$ in $E \backslash F_{E}$.

Since $F_{E}$ is a maximal forest, the endpoints of $e$ must be connected by edges in $F_{E}$. Call $e$ plus these connecting edges $L$. Let $x$ be any configuration with $x(e)=0$, and $f(x)$ be a new configuration constructed as follows. For all $e^{\prime} \in L$, let $f\left(e^{\prime}\right)=1-x\left(e^{\prime}\right)$. For all $e^{\prime} \notin L$, let $f\left(e^{\prime}\right)=x\left(e^{\prime}\right)$. This 'flips' the value of $x\left(e^{\prime}\right)$ along all the edges of the cycle $e \cup L$.

Since the edges are flipped along a cycle, the parity of each node is unchanged. This map $f$ is $1-1$ and onto, and so the partition function conditioned on $y(e)=0$ 
equals that conditioned on $y(e)=1$. In other words, there is a exactly a $1 / 2$ chance that $Y(e)=1$ for a draw $Y \sim \pi_{\text {subs }}$.

This holds even when conditioned on the values of all other edges in $E \backslash\left(F_{E} \cup\{e\}\right)$, since only edges in $F_{E}$ plus $e$ were used to construct $L$. Combining the $Z(e)=0$ and $Z(e)=1$ cases, the result is that $Y(e) \sim \operatorname{Bern}(Z(e) / 2)$, even when conditioned on the value of $Y\left(e^{\prime}\right)$ for all other edges $e^{\prime} \neq e$ not in $F_{E}$. Hence lines 3 through 5 of the algorithm are correct.

Now consider edge $e=\{i, j\}$ where $i$ is a leaf of $F_{E}$. Then $Y(e)$ must be chosen in such a way that the degree of $i$ in $Y$ is even. Line 9 accomplishes this task. This edge has been assigned a value, and can now be removed from $F_{E}$. As long as $F_{E}$ is nonempty, there will be at least two leaves left, so the process can be continued until the edges of $F_{E}$ are all assigned values by $Y$.

Note that the maximal forest $F_{E}$ can be constructed using either breadth first or depth first search. Either way, reversing the order in which nodes were added to the forest provides a way of recovering the leaves one by one with an overall running time that is linear in the size of the graph.

\section{Further notes}

\subsection{Proof of Theorem 1}

The reduction from subgraphs to random clusters as shown in Theorem 3 together with the reduction from random clusters to spins provides an immediate proof of Theorem 1 .

Proof of Theorem 1, Let $Z$ be a random cluster draw, and $X$ the spins draw that is obtained by uniformly at random choosing spin up or down for each cluster in $Z$. For a spins configuration $x$, let $S(x)$ denote the set of random cluster configurations that are consistent with $x$, so $z \in S(x)$ means for all $\{i, j\} \in E, x(i)=x(j)$ implies $z(\{i, j\})=1$. Then

$$
\begin{aligned}
\mathbb{P}(X=x) & =\sum_{z \in S(x)} \mathbb{P}(X=x \mid Z=z) \mathbb{P}(Z=z) \\
& =\sum_{z \in S(x)}(1 / 2)^{\# \mathcal{C}(z)}\left[\prod_{e: z(e)=1} p(e)\right]\left[\prod_{e: z(e)=0}(1-p(e))\right] 2^{\# \mathcal{C}(z)} Z_{\mathrm{rc}}^{-1} \\
& =Z_{\mathrm{rc}}^{-1} \prod_{e} \sum_{z(e) \leq \mathbf{1}(x(i)=x(j))} p(e) z(e)+(1-p(e))(1-z(e)) \\
& =Z_{\mathrm{rc}}^{-1} \prod_{e} 1 \cdot \mathbf{1}(x(i)=x(j))+(1-p(e)) \cdot \mathbf{1}(x(i) \neq x(j)) .
\end{aligned}
$$

Now $1-p(e)=0$ if $\beta(e)=\infty$. When $\beta(e)<\infty, 1-p(e)=\exp (-2 \beta(e))$. This means that for all $\beta(e)$ :

$$
\mathbf{1}(x(i)=x(j))+(1-p(e)) \mathbf{1}(x(i) \neq x(j))=f(x(i), x(j)) \exp (-\beta(e))
$$


where $\exp (-\infty)$ is taken to be 0 , and $f$ is as in (1). Hence

$$
\mathbb{P}(X=x)=Z_{\mathrm{rc}}^{-1} \prod_{e}[f(x(i), x(j)) \exp (-\beta(e))],
$$

which means that $Z_{\text {spins }}=Z_{\mathrm{rc}} \prod_{e} \exp (\beta(e))$.

Combining with Theorem 3 yields

$$
\begin{aligned}
Z_{\text {spins }} & =Z_{\text {subs }} 2^{\# V-\# E}\left[\prod_{e}(1+\exp (-2 \beta(e)))\right]\left[\prod_{e} \exp (\beta(e))\right] \\
& =Z_{\text {subs }} 2^{\# V} \prod_{e} \cosh \beta(e)
\end{aligned}
$$

as desired.

\subsection{Perfect simulation of subgraphs}

One of the original applications of the coupling from the past algorithm [6] was to generate samples perfectly from the random cluster model. Given the reduction of the previous section, this immediately gives the first perfect simulation method for the subgraphs world, which could prove useful in studying the model.

\section{References}

[1] J. Besag. Spatial interaction and the statistical analysis of lattice systems (with discussion). J. R. Statist. Soc. B, 36:192-236, 1974.

[2] C.M. Fortuin and P.W. Kasteleyn. On the random cluster model I: Introduction and relation to other models. Phys., 57:536-564, 1972.

[3] M. Jerrum and A. Sinclair. Polynomial-time approximation algorithms for the Ising model. SIAM J. Comput., 22:1087-1116, 1993.

[4] M. Jerrum, L. Valiant, and V. Vazirani. Random generation of combinatorial structures from a uniform distribution. Theoret. Comput. Sci., 43:169-188, 1986.

[5] G.F. Newell and E.W. Montroll. On the theory of the Ising model of ferromagnetism. Rev. Modern Phys., 25:353-389, 1953.

[6] J. G. Propp and D. B. Wilson. Exact sampling with coupled Markov chains and applications to statistical mechanics. Random Structures Algorithms, 9(1-2):223252, 1996.

[7] D. Randall and D. Wilson. Sampling spin configurations of an Ising system. In Proc. 10th ACM-SIAM Sympos. on Discrete Algorithms, pages 959-960, 1999.

[8] B. Simon. The Statistical Mechanics of Lattice Gasses, volume 1. Princeton University Press, 1993.

[9] R. Swendsen and J-S. Wang. Non-universal critical dynamics in Monte Carlo simulation. Phys. Rev. Lett., 58:86-88, 1987.

[10] B. L. van der Waerden. Die lang Reichweite der regelmäßigen Atomanordnung in Mischkristallen. Z. Physik, 118:473-488, 1941. 